\title{
Cardiac hypertrophy in Cushing's syndrome: if not hypertension then what?
}

\author{
D.A. Vassiliadi ${ }^{1} \cdot$ S. Tsagarakis ${ }^{1}$
}

Received: 2 November 2016 / Accepted: 4 February 2017 / Published online: 17 February 2017

(C) Springer Science+Business Media New York 2017

Cushing' s syndrome (CS) and hypertension share a special relationship that dates back to the beginning of the 20th century. It was Harvey Cushing who, by bringing RivaRocci's sphygmomanometer to his operating room, first introduced the systolic blood pressure measurement, which subsequently became one of the most important vital signs and a routine part of medical visits. It was also Harvey Cushing who, a decade later, first linked raised blood pressure to the clinical syndrome that now bears his name. Currently, it is acknowledged that hypertension is a prominent feature of CS affecting around $75 \%$ of the patients [1]. Cardiovascular disease is a major cause of morbidity and is largely responsible for the increased mortality of CS [2]. Importantly, the increased cardiovascular mortality risk in patients with Cushing's disease persists even after longterm remission [3, 4], suggesting that not only functional but also structural abnormalities develop that may not be reversible. Characterization and elucidation of the pathophysiological mechanisms behind the increased cardiovascular disease burden may result in proper diagnosis and treatment of this adverse outcome. To this end, it is quite surprising that only a relatively small number of studies have addressed this issue.

The existing evidence, mainly from studies using echocardiography [5-8], suggests that patients with CS have increased prevalence of left ventricular (LV) hypertrophy, higher frequency of concentric remodeling, and LV systolic

S. Tsagarakis

stsagara@otenet.gr

1 Department of Endocrinology, Diabetes and Metabolism, Evangelismos Hospital, 10676 Athens, Greece and diastolic dysfunction. LV mass index, however, is likely to be overestimated in echo-based studies. In a study that applied cardiac magnetic resonance (CMR) [9], subclinical biventricular and left atrium (LA) systolic dysfunction, as well as mainly regional LV wall thickness were found, although clinical LV hypertrophy according to the CMR criteria was detected in only one of the 18 patients. One study using ECG detected subtle abnormalities, LV hypertrophy and prolonged QT-corrected dispersion [10]. Despite the reported structural changes, LV ejection fraction is usually preserved and symptomatic heart failure is an uncommon presenting feature of CS. Nevertheless, the increased LV mass and the concentric LV remodeling are independent risk factors for overt heart failure and major cardiovascular events. Of note, some of these abnormalities, such as circumferential and longitudinal LV hypertrophy, biventricular systolic function, diastolic LV dysfunction and LA systolic dysfunction, are reversible after treatment $[8,9]$. However, the pathophysiology of these heart abnormalities remains largely elusive.

Hypertension is a well-established factor that promotes ventricular hypertrophy and confers risk for cardiovascular morbidity and mortality. Although a plausible cause, hypertension can only partly explain the observed morphological and functional heart changes in CS. Hypertension is not invariably present in CS and, also, LV hypertrophy has been described in patients without raised blood pressure [5, 6]. Most of the studies used single office or at home blood pressure recordings to define hypertension, and rather less attention has been paid to the overall daily pressure load. Single BP measurements at the clinic or at home may lead to overestimation ("white-coat hypertension") or underestimation ("masked hypertension") of BP levels, respectively, compared to ambulatory blood pressure 
monitoring [11]. It is increasingly recognized that the circadian profile of blood pressure may be important in determining individual cardiovascular risk and possibly affect heart remodeling [12]. Several patterns have been identified with a special focus on whether a person experiences the normal nocturnal fall (dip) of blood pressure $[13,14]$.

Avenatti et al. in their paper published in this issue of Endocrine [15], aimed to evaluate the possibility that the 24-h pressure load or the dipping status could be responsible for the observed adverse effects of CS on cardiac remodeling, using transthoracic echocardiography, and 24-h pressure monitoring. Overall, 25 patients with CS and 25 controls were included (9 normotensive and 16 hypertensive at each group). Although the number of participants is rather small, the analysis confirmed the presence of increased LV mass and relative wall thickness in CS patients compared to matched controls. The main finding of the study is that the actual pressure load or the dipping status of the patients could not explain the observed cardiac remodeling. These changes, also, did not correlate with the cortisol levels. This is in agreement with previous reports that relate the presence of cardiac hypertrophy more to the duration of hypercortisolism [6] and not to the severity of cortisol excess, indicating that this is a progressive process developing over time. Overall, this study adds to the scenario that the observed heart abnormalities in patients with CS are not directly related to blood pressure, and other contributing factors have to be considered. In this line, the reversibility of the structural changes did not relate to changes in blood pressure after treatment in two studies [8, 9] and, also, hypertension does not seem to be an independent risk factor for long-term mortality in Cushing's disease [4].

Besides hypertension, patients who are chronically exposed to elevated cortisol levels develop a constellation of factors known as metabolic syndrome, including central obesity, insulin resistance, hyperglycaemia and dyslipidaemia [7]. All these factors have negative impact on the heart, and may promote ventricular hypertrophy, fibrosis and deposition of fat. In fact, a recent study by Kamenicky et al. [9] reported that reversibility of some of these cardiac abnormalities after treatment of cortisol excess was, at least partly, related to the improvement of blood glucose levels and BMI.

Apart from the contribution of metabolic factors, a direct effect of cortisol should not be overlooked. The cardiovascular system is a central target of cortisol. Cortisol acts to maintain vascular tone and blood pressure in stressful situations associated with circulatory collapse. What is adaptive and beneficial in the event of stress may become disadvantageous in cases of cortisol excess, such as exogenous administration of glucocorticoids or CS.
The pathophysiology of the direct cortisol action on the cardiovascular system, albeit important, is much less comprehended, mainly due to its complexity [16]. Cortisol acts through both the glucocorticoid receptor-GR, which is ubiquitously expressed, and the mineralocorticoid receptor$\mathrm{MR}$, which is expressed in various tissues, including the myocardium, endothelial cells and the vascular smooth muscle. The relative abundance of GR and MR can differ according to tissue type. The action of cortisol on each receptor is regulated locally by pre-receptor modification by $11 \beta$-Hydroxysteroid dehydrogenase types 1 (HSD1) and 2 (HSD2). The HSD2 enzyme inactivates cortisol so that aldosterone (that circulates in much lower concentrations) can access the MR. In the myocardium, it has been postulated that local HSD2 is insufficient to inactivate cortisol that prevails over aldosterone for the MR and exerts diverse effects through both receptors. MR-mediated myocardial fibrosis, may thus be an important factor, but the data is contradictory, probably due to the use of different methods to assess fibrosis [9, 17]. Several other mechanisms include: induction of perivascular inflammation through activation of the MR, production of cytokines that may promote myocardial stiffness and contractile dysfunction by local inflammatory cells mainly the macrophages, reduced expression of eNOS and, potentiation of adrenergic action. Most of the data, however, come from ex vivo experiments or animal models making it difficult to delineate the contribution of each factor [16].

In conclusion, based on the data provided by Avennati et al [15], it is most likely that glucocorticoid excess induces adverse structural and functional cardiovascular changes that cannot be explained by the development of hypertension per se. Combined systemic and local effects of hypercortisolism may be the culprits for the development of cardiac hypertrophy through diverse mechanisms that are complex and thus hard to be fully elucidated. To this end, more focused studies using objective and reproducible methods for the assessment of cardiac function are required. From a clinical point of view, whatever the mechanisms, the consistently reported increased prevalence of an unfavorable cardiac remodeling should prompt routine evaluation of cardiovascular risk factors, and use of echocardiography in the assessment and follow-up of patients with CS. Furthermore, although the aggressive treatment of comorbidities like hypertension is a wise option in all patients with CS, the ongoing challenge of successfully controlling cortisol excess seems to be the safest and most effective option.

\section{Compliance with ethical standards}

Conflict of interest The authors declare that they have no competing interests. 
Ethical approval This is an invited Editorial, and does not contain any studies with human participants or animals performed by any of the authors.

\section{References}

1. J. Newell-Price, X. Bertagna, A.B. Grossman, L.K. Nieman, Cushing's syndrome. Lancet 367(9522), 1605-1617 (2006)

2. R.N. Clayton, D. Raskauskiene, R.C. Reulen, P.W. Jones, Mortality and morbidity in cushing's disease over 50 years in stoke-on-trent, UK: audit and meta-analysis of literature. J. Clin. Endocrinol. Metab. 96(3), 632-642 (2011)

3. F.M. van Haalen, L.H. Broersen, J.O. Jorgensen, A.M. Pereira, O.M. Dekkers, Management of endocrine disease: mortality remains increased in Cushing's disease despite biochemical remission: a systematic review and meta-analysis. Eur. J. Endocrinol. 172(4), R143-R149 (2015)

4. R.N. Clayton, P.W. Jones, R.C. Reulen, P.M. Stewart, Z.K. Hassan-Smith, G. Ntali, N. Karavitaki, O.M. Dekkers, A.M. Pereira, M. Bolland, I. Holdaway, J. Lindholm, Mortality in patients with Cushing's disease more than 10 years after remission: a multicentre, multinational, retrospective cohort study. Lancet Diabetes Endocrinol. 4(7), 569-576 (2016)

5. P.M. Toja, G. Branzi, F. Ciambellotti, P. Radaelli, M. De Martin, L.M. Lonati, M. Scacchi, G. Parati, F. Cavagnini, F. Pecori Giraldi, Clinical relevance of cardiac structure and function abnormalities in patients with Cushing's syndrome before and after cure. Clin. Endocrinol. 76(3), 332-338 (2012)

6. M.L. Muiesan, M. Lupia, M. Salvetti, C. Grigoletto, N. Sonino, M. Boscaro, E.A. Rosei, F. Mantero, F. Fallo, Left ventricular structural and functional characteristics in Cushing's syndrome. J. Am. Coll. Cardiol. 41(12), 2275-2279 (2003)

7. A.M. Isidori, C. Graziadio, R.M. Paragliola, A. Cozzolino, A.G. Ambrogio, A. Colao, S.M. Corsello, R. Pivonello; Group ABCS, The hypertension of Cushing's syndrome: controversies in the pathophysiology and focus on cardiovascular complications. J. Hypertens. 33(1), 44-60 (2015)

8. A.M. Pereira, V. Delgado, J.A. Romijn, J.W. Smit, J.J. Bax, R.A. Feelders, Cardiac dysfunction is reversed upon successful treatment of Cushing's syndrome. Eur. J. Endocrinol. 162(2), 331-340 (2010)

9. P. Kamenicky, A. Redheuil, C. Roux, S. Salenave, N. Kachenoura, Z. Raissouni, L. Macron, L. Guignat, C. Jublanc, A. Azarine, S. Brailly, J. Young, E. Mousseaux, P. Chanson, Cardiac structure and function in Cushing's syndrome: a cardiac magnetic resonance imaging study. J. Clin. Endocrinol. Metab. 99 (11), E2144-E2153 (2014)

10. K.I. Alexandraki, G.A. Kaltsas, A.I. Vouliotis, T.G. Papaioannou, L. Trisk, A. Zilos, M. Korbonits, G.M. Besser, A. Anastasakis, A.B. Grossman, Specific electrocardiographic features associated with Cushing's disease. Clin. Endocrinol. 74(5), 558-564 (2011)

11. Y.Y. Kang, Y. Li, Q.F. Huang, J. Song, X.L. Shan, Y. Dou, X.J. $\mathrm{Xu}$, S.H. Chen, J.G. Wang, Accuracy of home versus ambulatory blood pressure monitoring in the diagnosis of white-coat and masked hypertension. J. Hypertens. 33(8), 1580-1587 (2015)

12. J.A. Staessen, L. Thijs, R. Fagard, E.T. O'Brien, D. Clement, P.W. de Leeuw, G. Mancia, C. Nachev, P. Palatini, G. Parati, J. Tuomilehto, J. Webster, Predicting cardiovascular risk using conventional vs ambulatory blood pressure in older patients with systolic hypertension. systolic hypertension in Europe trial investigators. JAMA 282(6), 539-546 (1999)

13. P. Verdecchia, G. Schillaci, M. Guerrieri, C. Gatteschi, G. Benemio, F. Boldrini, C. Porcellati, Circadian blood pressure changes and left ventricular hypertrophy in essential hypertension. Circulation 81(2), 528-536 (1990)

14. T.W. Hansen, Y. Li, J. Boggia, L. Thijs, T. Richart, J.A. Staessen, Predictive role of the nighttime blood pressure. Hypertension 57(1), 3-10 (2011)

15. E. Avenatti, A. Rebellato, A. Iannaccone, M. Battocchio, F. Dassie, F. Veglio, A. Milan, F. Fallo, Left ventricular geometry and 24-h blood pressure profile in Cushing's syndrome. Endocrine 55(2), 547-554 (2017)

16. B.R. Walker, Glucocorticoids and cardiovascular disease. Eur. J. Endocrinol. 157(5), 545-559 (2007)

17. K.H. Yiu, N.A. Marsan, V. Delgado, N.R. Biermasz, E.R. Holman, J.W. Smit, R.A. Feelders, J.J. Bax, A.M. Pereira, Increased myocardial fibrosis and left ventricular dysfunction in Cushing's syndrome. Eur. J. Endocrinol. 166(1), 27-34 (2012) 